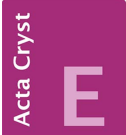
COMMUNICATIONS

ISSN 2056-9890

\section{Crystal structure of 1-(cyclopentylidene- amino)-3-(prop-2-en-1-yl)thiourea}

Shaaban K. Mohamed, ${ }^{a, b}$ Joel T. Mague, ${ }^{\text {c }}$ Mehmet Akkurt, ${ }^{\text {d }}$ Alaa A. Hassan, ${ }^{b}$ Ahmed T. Abdel-Aziz ${ }^{b}$ and Mustafa R. Albayati $^{\mathbf{e}_{*}}$

${ }^{\text {a } F a c u l t y ~ o f ~ S c i e n c e ~ \& ~ E n g i n e e r i n g, ~ S c h o o l ~ o f ~ H e a l t h c a r e ~ S c i e n c e, ~ M a n c h e s t e r ~}$ Metropolitan University, Manchester M1 5GD, England, 'bChemistry Department, Faculty of Science, Minia University, 61519 El-Minia, Egypt, 'Department of Chemistry, Tulane University, New Orleans, LA 70118, USA, ${ }^{\mathbf{d} D e p a r t m e n t}$ of Physics, Faculty of Sciences, Erciyes University, 38039 Kayseri, Turkey, and ${ }^{{ }^{2} K i r k u k}$ University, College of Education, Department of Chemistry, Kirkuk, Iraq. *Correspondence e-mail: shaabankamel@yahoo.com

Received 3 November 2015; accepted 5 November 2015

Edited by W. T. A. Harrison, University of Aberdeen, Scotland

In the title compound, $\mathrm{C}_{9} \mathrm{H}_{15} \mathrm{~N}_{3} \mathrm{~S}$, the cyclopentyl ring adopts an envelope conformation with one of the methylene $\mathrm{C}$ atoms as the flap. The thiosemicarbazide fragment is almost planar (r.m.s. deviation $=0.038 \AA$ ) and a short intramolecular $\mathrm{N}-$ $\mathrm{H} \cdot \mathrm{N}$ contact occurs. In the crystal, molecules are linked into helical $\left(4_{1}\right.$ symmetry) chains propagating in [001] by $\mathrm{N}-$ $\mathrm{H} \cdots \mathrm{N}$ and $\mathrm{N}-\mathrm{H} \cdots \mathrm{S}$ hydrogen bonds. A very weak $\mathrm{C}-\mathrm{H} \cdots \mathrm{S}$ interaction is also observed.

Keywords: crystal structure; thiosemicarbazides; hydrogen bonding.

CCDC reference: 1435175

\section{Related literature}

For the biological activities of thiosemicarbazide-containing compounds, see: Hu et al. (2010); da Costa et al. (2015). For the synthesis of the title compound, see: Mague et al. (2014).

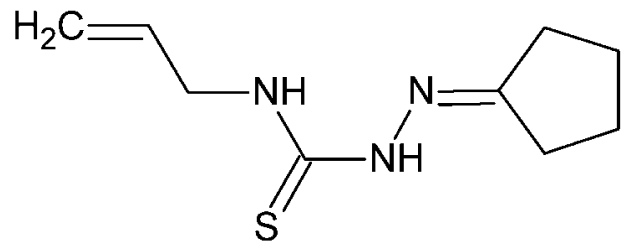

\section{Experimental}

2.1. Crystal data

$\mathrm{C}_{9} \mathrm{H}_{15} \mathrm{~N}_{3} \mathrm{~S}$

$M_{r}=197.30$
Tetragonal, $P 4_{1}$

$a=9.0124(2) \AA$

$c=12.8200(2) \AA$

$V=1041.28(5) \AA^{3}$

$Z=4$

\subsection{Data collection \\ Bruker D8 VENTURE PHOTON 100 CMOS diffractometer \\ Absorption correction: multi-scan (SADABS; Bruker, 2014) \\ $T_{\min }=0.67, T_{\max }=0.79$}

\subsection{Refinement}

$R\left[F^{2}>2 \sigma\left(F^{2}\right)\right]=0.041$

$w R\left(F^{2}\right)=0.109$

$S=1.10$

1990 reflections

119 parameters

1 restraint

$\mathrm{H}$-atom parameters constrained

$\Delta \rho_{\max }=0.70 \mathrm{e}^{-3}$

$\Delta \rho_{\min }=-0.18$ e $\AA^{-3}$

Absolute structure: Flack $x$ determined using 826 quotients $\left[\left(I^{+}\right)-\left(I^{-}\right)\right] /\left[\left(I^{+}\right)+\left(I^{-}\right)\right]$(Parsons et al., 2013)

Absolute structure parameter: 0.04 (3)

Table 1

Hydrogen-bond geometry $\left(\AA,^{\circ}\right)$.

\begin{tabular}{lllll}
\hline$D-\mathrm{H} \cdots A$ & $D-\mathrm{H}$ & $\mathrm{H} \cdots A$ & $D \cdots A$ & $D-\mathrm{H} \cdots A$ \\
\hline $\mathrm{N} 2-\mathrm{H} 2 C \cdots \mathrm{N} 1^{\mathrm{i}}$ & 0.91 & 2.29 & $3.194(4)$ & 177 \\
$\mathrm{~N} 3-\mathrm{H} 3 C \cdots \mathrm{N} 1$ & 0.91 & 2.25 & $2.642(4)$ & 106 \\
$\mathrm{~N} 3-\mathrm{H} 3 C \cdots \mathrm{S} 1^{\mathrm{ii}}$ & 0.91 & 2.49 & $3.310(3)$ & 151 \\
$\mathrm{C} 3-\mathrm{H} 3 A \cdots \mathrm{S} 1^{\mathrm{iii}}$ & 0.99 & 2.86 & $3.663(4)$ & 139 \\
\hline
\end{tabular}

Symmetry codes: (i) $-y+1, x, z+\frac{1}{4}$; (ii) $y,-x+1, z-\frac{1}{4}$; (iii) $-y, x, z+\frac{1}{4}$.

Data collection: APEX2 (Bruker, 2014); cell refinement: SAINT (Bruker, 2014); data reduction: $S A I N T$; $\operatorname{program}(\mathrm{s})$ used to solve structure: SHELXT (Sheldrick, 2015a); program(s) used to refine structure: SHELXL2014 (Sheldrick, 2015b); molecular graphics: DIAMOND (Brandenburg \& Putz, 2012); software used to prepare material for publication: SHELXTL (Sheldrick, 2008).

\title{
Acknowledgements
}

The support of NSF-MRI Grant No. 1228232 for the purchase of the diffractometer and Tulane University for support of the Tulane Crystallography Laboratory are gratefully acknowledged.

Supporting information for this paper is available from the IUCr electronic archives (Reference: HB7535).

\section{References}

Brandenburg, K. \& Putz, H. (2012). DIAMOND. Crystal Impact GbR, Bonn, Germany.

Bruker (2014). APEX2, SAINT and SADABS. Bruker AXS Inc., Madison, Wisconsin, USA.

Costa, P. M. da, da Costa, M. P., Carvalho, A. A., Cavalcanti, M. S., de Oliveira Cardoso, M. V., de Oliveira Filho, G. B., de Araújo Viana, D., FechineJamacaru, F. V., Leite, A. C., de Moraes, M. O., Pessoa, C. \& Ferreira, P. M. (2015). Chem. Biol. Interact. 239, 174-183.

Hu, K., Yang, Z., Pan, S., Xu, H. \& Ren, J. (2010). Eur. J. Med. Chem. 45, $3453-$ 3458. 
Mague, J. T., Mohamed, S. K., Akkurt, M., Hassan, A. A. \& Albayati, M. R. (2014). Acta Cryst. E70, o515.

Parsons, S., Flack, H. D. \& Wagner, T. (2013). Acta Cryst. B69, 249-259.
Sheldrick, G. M. (2008). Acta Cryst. A64, 112-122.

Sheldrick, G. M. (2015a). Acta Cryst. A71, 3-8.

Sheldrick, G. M. (2015b). Acta Cryst. C71, 3-8. 


\section{supporting information}

Acta Cryst. (2015). E71, o924-o925 [https://doi.org/10.1107/S2056989015021003]

\section{Crystal structure of 1-(cyclopentylideneamino)-3-(prop-2-en-1-yl)thiourea}

Shaaban K. Mohamed, Joel T. Mague, Mehmet Akkurt, Alaa A. Hassan, Ahmed T. Abdel-Aziz and Mustafa R. Albayati

\section{S1. Comment}

The thiosemicarbazones comprise a class of molecules known for their diverse biological activities (Hu et al., 2010; Costa et al., 2015). In this context we report here the synthesis and crystal structure determination of the title compound. In the title compound (Fig. 1), a Cremer-Pople analysis of the conformation of the 5-membered ring gave puckering parameters $\mathrm{Q}(2)=0.380$ (4) $\AA$ and $\varphi(2)=285.3(6)^{\circ}$. The molecules pack in helical chains about the $4_{1}$ axis assisted by an $\mathrm{N} 2-\mathrm{H} 2 \mathrm{C} \cdots \mathrm{N} 1^{\mathrm{i}}$ and an $\mathrm{N} 3-\mathrm{H} 3 \mathrm{C} \cdots \mathrm{S} 1^{\mathrm{ii}}(\mathrm{i}: 1-y, x, 1 / 4+z$; ii: $y, 1-x,-1 / 4+z)$ hydrogen bond between each pair of adjacent molecules (Table 1 and Fig. 2).

\section{S2. Experimental}

The title compound was prepared according to our recently reported method (Mague et al., 2014). Colourless blocks were grown from ethanol solution by slow evaporation. M. p. 374-375 K; 89\% yield.

\section{S3. Refinement}

$\mathrm{H}$ atoms attached to carbon were placed in calculated positions $(\mathrm{C}-\mathrm{H}=0.95-0.99 \AA)$ while those attached to nitrogen were placed in locations derived from a difference map and their parameters adjusted to give $\mathrm{N}-\mathrm{H}=0.91 \AA$. All were included as riding contributions with isotropic displacement parameters 1.2 times those of the attached atoms.

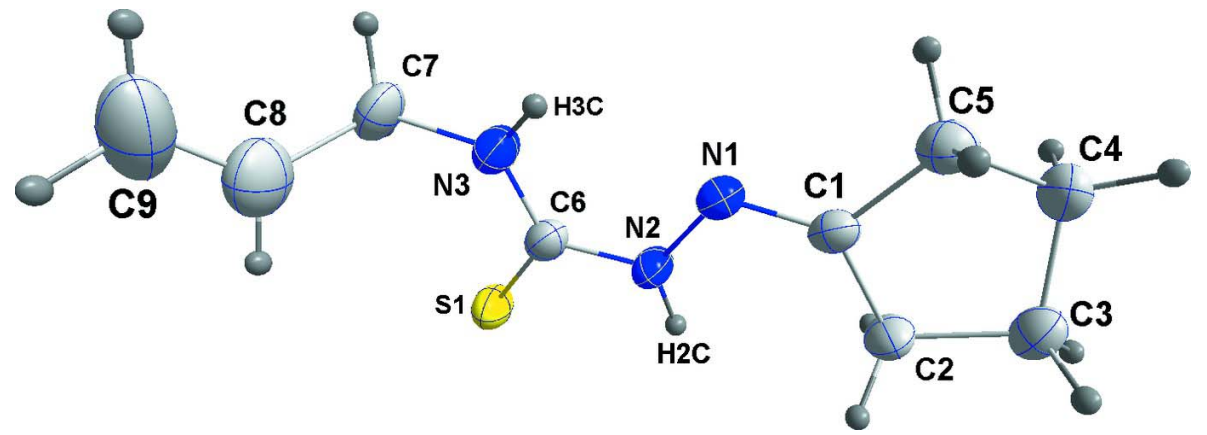

Figure 1

The title molecule, shown with $50 \%$ probability ellipsoids. 


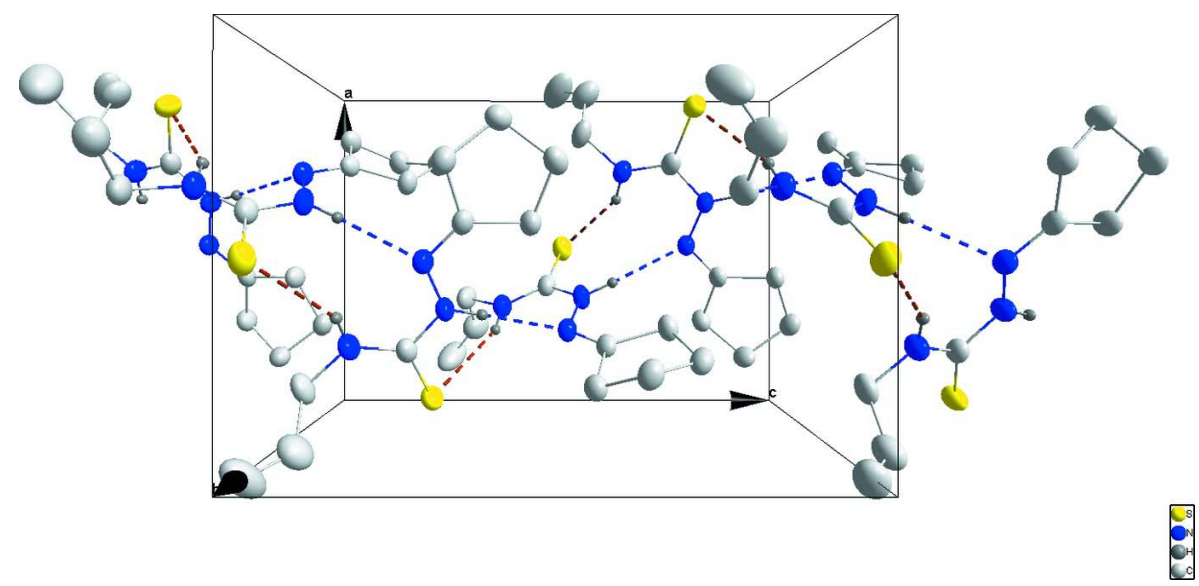

Figure 2

The packing in the title molecule, viewed down the $b$ axis. $\mathrm{N}-\mathrm{H} \cdots \mathrm{N}$ and $\mathrm{N}-\mathrm{H} \cdots \mathrm{S}$ hydrogen bonds are shown, respectively, as blue and brown dotted lines.

1-(Cyclopentylideneamino)-3-(prop-2-en-1-yl)thiourea

\section{Crystal data}

\section{$\mathrm{C}_{9} \mathrm{H}_{15} \mathrm{~N}_{3} \mathrm{~S}$}

$M_{r}=197.30$

Tetragonal, $P 4_{1}$

Hall symbol: P $4 \mathrm{w}$

$a=9.0124(2) \AA$

$c=12.8200(2) \AA$

$V=1041.28(5) \AA^{3}$

$Z=4$

$F(000)=424$

\section{Data collection}

\section{Bruker D8 VENTURE PHOTON 100 CMOS} diffractometer

Radiation source: INCOATEC I $\mu \mathrm{S}$ micro-focus source

Mirror monochromator

Detector resolution: 10.4167 pixels $\mathrm{mm}^{-1}$

$\omega$ scans

Absorption correction: multi-scan

(SADABS; Bruker, 2014)

\section{Refinement}

Refinement on $F^{2}$

Least-squares matrix: full

$R\left[F^{2}>2 \sigma\left(F^{2}\right)\right]=0.041$

$w R\left(F^{2}\right)=0.109$

$S=1.10$

1990 reflections

119 parameters

1 restraint

Hydrogen site location: mixed

$\mathrm{H}$-atom parameters constrained
$D_{\mathrm{x}}=1.259 \mathrm{Mg} \mathrm{m}^{-3}$

$\mathrm{Cu} K \alpha$ radiation, $\lambda=1.54178 \AA$

Cell parameters from 7005 reflections

$\theta=3.5-72.4^{\circ}$

$\mu=2.42 \mathrm{~mm}^{-1}$

$T=150 \mathrm{~K}$

Block, colourless

$0.21 \times 0.16 \times 0.10 \mathrm{~mm}$

$T_{\min }=0.67, T_{\max }=0.79$

7869 measured reflections

1990 independent reflections

1901 reflections with $I>2 \sigma(I)$

$R_{\text {int }}=0.032$

$\theta_{\max }=72.4^{\circ}, \theta_{\min }=4.9^{\circ}$

$h=-10 \rightarrow 11$

$k=-11 \rightarrow 11$

$l=-15 \rightarrow 15$

$w=1 /\left[\sigma^{2}\left(F_{\mathrm{o}}^{2}\right)+(0.0616 P)^{2}+0.3211 P\right]$ where $P=\left(F_{\mathrm{o}}^{2}+2 F_{\mathrm{c}}^{2}\right) / 3$

$(\Delta / \sigma)_{\max }<0.001$

$\Delta \rho_{\max }=0.70 \mathrm{e} \AA^{-3}$

$\Delta \rho_{\min }=-0.18 \mathrm{e} \AA^{-3}$

Absolute structure: Flack $x$ determined using 826 quotients $\left[\left(I^{+}\right)-\left(I^{-}\right)\right] /\left[\left(I^{+}\right)+\left(I^{-}\right)\right]$(Parsons et al., 2013)

Absolute structure parameter: 0.04 (3) 


\section{Special details}

Geometry. Bond distances, angles etc. have been calculated using the rounded fractional coordinates. All su's are estimated from the variances of the (full) variance-covariance matrix. The cell e.s.d.'s are taken into account in the estimation of distances, angles and torsion angles

Refinement. Refinement on $F^{2}$ for ALL reflections except those flagged by the user for potential systematic errors. Weighted $R$-factors $w R$ and all goodnesses of fit $S$ are based on $F^{2}$, conventional $R$-factors $R$ are based on $F$, with $F$ set to zero for negative $F^{2}$. The observed criterion of $F^{2}>\sigma\left(F^{2}\right)$ is used only for calculating - $R$-factor-obs etc. and is not relevant to the choice of reflections for refinement. $R$-factors based on $F^{2}$ are statistically about twice as large as those based on $F$, and $R$-factors based on ALL data will be even larger.

Fractional atomic coordinates and isotropic or equivalent isotropic displacement parameters $\left(\AA^{2}\right)$

\begin{tabular}{|c|c|c|c|c|}
\hline & $x$ & $y$ & $z$ & $U_{\text {iso }} * / U_{\text {eq }}$ \\
\hline S1 & $0.50830(11)$ & $0.10489(10)$ & $0.51268(7)$ & $0.0404(3)$ \\
\hline N1 & $0.2886(3)$ & $0.4807(3)$ & $0.5217(2)$ & $0.0312(8)$ \\
\hline N2 & $0.3701(3)$ & $0.3575(3)$ & $0.5510(2)$ & $0.0325(8)$ \\
\hline N3 & $0.3362(3)$ & $0.2660(3)$ & $0.3854(2)$ & $0.0357(9)$ \\
\hline $\mathrm{C} 1$ & $0.2584(4)$ & $0.5764(4)$ & $0.5926(3)$ & $0.0293(9)$ \\
\hline $\mathrm{C} 2$ & $0.3050(4)$ & $0.5761(4)$ & $0.7060(3)$ & $0.0339(10)$ \\
\hline C3 & $0.2402(4)$ & $0.7223(4)$ & 0.7498 & $0.0362(10)$ \\
\hline $\mathrm{C} 4$ & $0.2242(4)$ & $0.8220(4)$ & $0.6547(3)$ & $0.0389(11)$ \\
\hline $\mathrm{C} 5$ & $0.1737(4)$ & $0.7160(4)$ & $0.5686(3)$ & $0.0359(11)$ \\
\hline C6 & $0.3975(4)$ & $0.2491(4)$ & $0.4790(3)$ & $0.0331(10)$ \\
\hline C7 & $0.3533(5)$ & $0.1587(5)$ & $0.3012(3)$ & $0.0430(11)$ \\
\hline $\mathrm{C} 8$ & $0.2503(6)$ & $0.0262(6)$ & $0.3117(4)$ & $0.0617(17)$ \\
\hline C9 & $0.1462(7)$ & $-0.0066(7)$ & $0.2532(5)$ & $0.079(2)$ \\
\hline $\mathrm{H} 2 \mathrm{~A}$ & 0.41450 & 0.57460 & 0.71240 & $0.0410^{*}$ \\
\hline $\mathrm{H} 2 \mathrm{~B}$ & 0.26350 & 0.48910 & 0.74300 & $0.0410 *$ \\
\hline $\mathrm{H} 2 \mathrm{C}$ & 0.41060 & 0.34020 & 0.61480 & $0.0390^{*}$ \\
\hline $\mathrm{H} 3 \mathrm{~A}$ & 0.14260 & 0.70480 & 0.78310 & $0.0430 *$ \\
\hline H3B & 0.30820 & 0.76700 & 0.80170 & $0.0430 *$ \\
\hline $\mathrm{H} 3 \mathrm{C}$ & 0.27400 & 0.34430 & 0.37540 & $0.0430^{*}$ \\
\hline $\mathrm{H} 4 \mathrm{~A}$ & 0.32000 & 0.86910 & 0.63660 & $0.0470^{*}$ \\
\hline H4B & 0.14930 & 0.90050 & 0.66700 & $0.0470^{*}$ \\
\hline $\mathrm{H} 5 \mathrm{~A}$ & 0.06530 & 0.69880 & 0.57190 & $0.0430 *$ \\
\hline H5B & 0.19970 & 0.75490 & 0.49880 & $0.0430 *$ \\
\hline H7A & 0.45720 & 0.12310 & 0.30000 & $0.0520 *$ \\
\hline H7B & 0.33340 & 0.20860 & 0.23380 & $0.0520 *$ \\
\hline H8 & 0.26760 & -0.03810 & 0.36910 & $0.0740^{*}$ \\
\hline H9A & 0.12420 & 0.05410 & 0.19460 & $0.0950 *$ \\
\hline H9B & 0.08830 & -0.09250 & 0.26690 & $0.0950 *$ \\
\hline
\end{tabular}

Atomic displacement parameters $\left(\AA^{2}\right)$

\begin{tabular}{lllllll}
\hline & $U^{11}$ & $U^{22}$ & $U^{33}$ & $U^{12}$ & $U^{13}$ & $U^{23}$ \\
\hline S1 & $0.0529(6)$ & $0.0352(4)$ & $0.0332(4)$ & $0.0076(4)$ & $-0.0021(4)$ & $-0.0046(4)$ \\
N1 & $0.0366(14)$ & $0.0334(13)$ & $0.0235(12)$ & $-0.0002(11)$ & $-0.0003(12)$ & $0.0003(12)$ \\
N2 & $0.0433(16)$ & $0.0325(15)$ & $0.0217(12)$ & $0.0033(12)$ & $-0.0040(12)$ & $-0.0021(11)$ \\
N3 & $0.0413(17)$ & $0.0387(17)$ & $0.0272(15)$ & $0.0005(12)$ & $-0.0016(13)$ & $-0.0047(12)$
\end{tabular}




\begin{tabular}{lllllll} 
C1 & $0.0316(16)$ & $0.0328(17)$ & $0.0236(16)$ & $-0.0033(13)$ & $0.0013(13)$ & $0.0030(13)$ \\
C2 & $0.0415(19)$ & $0.0357(18)$ & $0.0245(17)$ & $0.0010(15)$ & $-0.0038(14)$ & $0.0002(13)$ \\
C3 & $0.0379(18)$ & $0.0401(18)$ & $0.0307(17)$ & $-0.0040(14)$ & $0.0039(15)$ & $-0.0051(15)$ \\
C4 & $0.047(2)$ & $0.0338(18)$ & $0.036(2)$ & $0.0042(15)$ & $0.0050(17)$ & $-0.0026(15)$ \\
C5 & $0.0389(18)$ & $0.0392(19)$ & $0.0297(18)$ & $0.0042(15)$ & $-0.0013(14)$ & $0.0033(15)$ \\
C6 & $0.0372(17)$ & $0.0361(18)$ & $0.0260(16)$ & $-0.0053(13)$ & $0.0023(14)$ & $-0.0018(13)$ \\
C7 & $0.044(2)$ & $0.055(2)$ & $0.0299(17)$ & $-0.0055(17)$ & $0.0020(15)$ & $-0.0131(17)$ \\
C8 & $0.065(3)$ & $0.061(3)$ & $0.059(3)$ & $-0.002(2)$ & $0.005(2)$ & $-0.018(2)$ \\
C9 & $0.075(3)$ & $0.090(4)$ & $0.073(4)$ & $-0.040(3)$ & $0.019(3)$ & $-0.028(3)$ \\
\hline
\end{tabular}

Geometric parameters $\left(\AA,{ }^{\circ}\right)$

\begin{tabular}{|c|c|c|c|}
\hline $\mathrm{S} 1-\mathrm{C} 6$ & $1.695(4)$ & $\mathrm{C} 8-\mathrm{C} 9$ & $1.237(8)$ \\
\hline $\mathrm{N} 1-\mathrm{N} 2$ & $1.383(4)$ & $\mathrm{C} 2-\mathrm{H} 2 \mathrm{~A}$ & 0.9900 \\
\hline $\mathrm{N} 1-\mathrm{C} 1$ & $1.282(5)$ & $\mathrm{C} 2-\mathrm{H} 2 \mathrm{~B}$ & 0.9900 \\
\hline $\mathrm{N} 2-\mathrm{C} 6$ & $1.367(5)$ & $\mathrm{C} 3-\mathrm{H} 3 \mathrm{~A}$ & 0.9900 \\
\hline N3-C6 & $1.330(5)$ & $\mathrm{C} 3-\mathrm{H} 3 \mathrm{~B}$ & 0.9900 \\
\hline $\mathrm{N} 3-\mathrm{C} 7$ & $1.457(5)$ & $\mathrm{C} 4-\mathrm{H} 4 \mathrm{~A}$ & 0.9900 \\
\hline $\mathrm{C} 1-\mathrm{C} 2$ & $1.513(5)$ & $\mathrm{C} 4-\mathrm{H} 4 \mathrm{~B}$ & 0.9900 \\
\hline $\mathrm{C} 1-\mathrm{C} 5$ & $1.503(5)$ & $\mathrm{C} 5-\mathrm{H} 5 \mathrm{~A}$ & 0.9900 \\
\hline $\mathrm{C} 2-\mathrm{C} 3$ & $1.547(5)$ & $\mathrm{C} 5-\mathrm{H} 5 \mathrm{~B}$ & 0.9900 \\
\hline $\mathrm{N} 2-\mathrm{H} 2 \mathrm{C}$ & 0.9100 & $\mathrm{C} 7-\mathrm{H} 7 \mathrm{~A}$ & 0.9900 \\
\hline $\mathrm{C} 3-\mathrm{C} 4$ & $1.521(5)$ & $\mathrm{C} 7-\mathrm{H} 7 \mathrm{~B}$ & 0.9900 \\
\hline $\mathrm{N} 3-\mathrm{H} 3 \mathrm{C}$ & 0.9100 & $\mathrm{C} 8-\mathrm{H} 8$ & 0.9500 \\
\hline $\mathrm{C} 4-\mathrm{C} 5$ & $1.529(5)$ & C9-H9A & 0.9500 \\
\hline $\mathrm{C} 7-\mathrm{C} 8$ & $1.519(7)$ & C9-H9B & 0.9500 \\
\hline $\mathrm{N} 2-\mathrm{N} 1-\mathrm{C} 1$ & $117.4(3)$ & $\mathrm{C} 2-\mathrm{C} 3-\mathrm{H} 3 \mathrm{~B}$ & 111.00 \\
\hline $\mathrm{N} 1-\mathrm{N} 2-\mathrm{C} 6$ & $119.1(3)$ & $\mathrm{C} 4-\mathrm{C} 3-\mathrm{H} 3 \mathrm{~A}$ & 111.00 \\
\hline $\mathrm{C} 6-\mathrm{N} 3-\mathrm{C} 7$ & $123.3(3)$ & $\mathrm{C} 4-\mathrm{C} 3-\mathrm{H} 3 \mathrm{~B}$ & 111.00 \\
\hline $\mathrm{N} 1-\mathrm{C} 1-\mathrm{C} 2$ & $128.4(3)$ & $\mathrm{H} 3 \mathrm{~A}-\mathrm{C} 3-\mathrm{H} 3 \mathrm{~B}$ & 109.00 \\
\hline $\mathrm{N} 1-\mathrm{C} 1-\mathrm{C} 5$ & $121.7(3)$ & $\mathrm{C} 3-\mathrm{C} 4-\mathrm{H} 4 \mathrm{~A}$ & 111.00 \\
\hline $\mathrm{C} 2-\mathrm{C} 1-\mathrm{C} 5$ & $109.8(3)$ & $\mathrm{C} 3-\mathrm{C} 4-\mathrm{H} 4 \mathrm{~B}$ & 111.00 \\
\hline $\mathrm{C} 1-\mathrm{C} 2-\mathrm{C} 3$ & $104.0(3)$ & $\mathrm{C} 5-\mathrm{C} 4-\mathrm{H} 4 \mathrm{~A}$ & 111.00 \\
\hline $\mathrm{N} 1-\mathrm{N} 2-\mathrm{H} 2 \mathrm{C}$ & 127.00 & $\mathrm{C} 5-\mathrm{C} 4-\mathrm{H} 4 \mathrm{~B}$ & 111.00 \\
\hline $\mathrm{C} 6-\mathrm{N} 2-\mathrm{H} 2 \mathrm{C}$ & 114.00 & $\mathrm{H} 4 \mathrm{~A}-\mathrm{C} 4-\mathrm{H} 4 \mathrm{~B}$ & 109.00 \\
\hline $\mathrm{C} 2-\mathrm{C} 3-\mathrm{C} 4$ & $104.4(3)$ & $\mathrm{C} 1-\mathrm{C} 5-\mathrm{H} 5 \mathrm{~A}$ & 111.00 \\
\hline $\mathrm{C} 6-\mathrm{N} 3-\mathrm{H} 3 \mathrm{C}$ & 118.00 & $\mathrm{C} 1-\mathrm{C} 5-\mathrm{H} 5 \mathrm{~B}$ & 111.00 \\
\hline $\mathrm{C} 7-\mathrm{N} 3-\mathrm{H} 3 \mathrm{C}$ & 118.00 & $\mathrm{C} 4-\mathrm{C} 5-\mathrm{H} 5 \mathrm{~A}$ & 111.00 \\
\hline $\mathrm{C} 3-\mathrm{C} 4-\mathrm{C} 5$ & $103.8(3)$ & $\mathrm{C} 4-\mathrm{C} 5-\mathrm{H} 5 \mathrm{~B}$ & 111.00 \\
\hline $\mathrm{C} 1-\mathrm{C} 5-\mathrm{C} 4$ & $102.9(3)$ & $\mathrm{H} 5 \mathrm{~A}-\mathrm{C} 5-\mathrm{H} 5 \mathrm{~B}$ & 109.00 \\
\hline $\mathrm{S} 1-\mathrm{C} 6-\mathrm{N} 2$ & $118.9(3)$ & $\mathrm{N} 3-\mathrm{C} 7-\mathrm{H} 7 \mathrm{~A}$ & 109.00 \\
\hline $\mathrm{N} 2-\mathrm{C} 6-\mathrm{N} 3$ & $116.9(3)$ & $\mathrm{N} 3-\mathrm{C} 7-\mathrm{H} 7 \mathrm{~B}$ & 109.00 \\
\hline $\mathrm{S} 1-\mathrm{C} 6-\mathrm{N} 3$ & $124.2(3)$ & $\mathrm{C} 8-\mathrm{C} 7-\mathrm{H} 7 \mathrm{~A}$ & 109.00 \\
\hline $\mathrm{N} 3-\mathrm{C} 7-\mathrm{C} 8$ & $113.1(3)$ & $\mathrm{C} 8-\mathrm{C} 7-\mathrm{H} 7 \mathrm{~B}$ & 109.00 \\
\hline $\mathrm{C} 7-\mathrm{C} 8-\mathrm{C} 9$ & $126.7(5)$ & $\mathrm{H} 7 \mathrm{~A}-\mathrm{C} 7-\mathrm{H} 7 \mathrm{~B}$ & 108.00 \\
\hline $\mathrm{C} 1-\mathrm{C} 2-\mathrm{H} 2 \mathrm{~A}$ & 111.00 & $\mathrm{C} 7-\mathrm{C} 8-\mathrm{H} 8$ & 117.00 \\
\hline $\mathrm{C} 1-\mathrm{C} 2-\mathrm{H} 2 \mathrm{~B}$ & 111.00 & $\mathrm{C} 9-\mathrm{C} 8-\mathrm{H} 8$ & 117.00 \\
\hline
\end{tabular}




$\begin{array}{llll}\mathrm{C} 3-\mathrm{C} 2-\mathrm{H} 2 \mathrm{~A} & 111.00 & \mathrm{C} 8-\mathrm{C} 9-\mathrm{H} 9 \mathrm{~A} & 120.00 \\ \mathrm{C} 3-\mathrm{C} 2-\mathrm{H} 2 \mathrm{~B} & 111.00 & \mathrm{C} 8-\mathrm{C} 9-\mathrm{H} 9 \mathrm{~B} & 120.00 \\ \mathrm{H} 2 \mathrm{~A}-\mathrm{C} 2-\mathrm{H} 2 \mathrm{~B} & 109.00 & \mathrm{H} 9 \mathrm{~A}-\mathrm{C} 9-\mathrm{H} 9 \mathrm{~B} & 120.00 \\ \mathrm{C} 2-\mathrm{C} 3-\mathrm{H} 3 \mathrm{~A} & 111.00 & & \\ & & & \\ \mathrm{C} 1-\mathrm{N} 1-\mathrm{N} 2-\mathrm{C} 6 & 176.4(3) & \mathrm{N} 1-\mathrm{C} 1-\mathrm{C} 2-\mathrm{C} 3 & 177.8(4) \\ \mathrm{N} 2-\mathrm{N} 1-\mathrm{C} 1-\mathrm{C} 2 & 2.3(5) & \mathrm{N} 1-\mathrm{C} 1-\mathrm{C} 2-\mathrm{C} 5-\mathrm{C} 3 & -154.9(3) \\ \mathrm{N} 2-\mathrm{N} 1-\mathrm{C} 1-\mathrm{C} 5 & 178.5(3) & \mathrm{C} 2-\mathrm{C} 1-\mathrm{C} 5-\mathrm{C} 4 & 21.9(4) \\ \mathrm{N} 1-\mathrm{N} 2-\mathrm{C} 6-\mathrm{S} 1 & 175.4(2) & \mathrm{C} 1-\mathrm{C} 2-\mathrm{C} 3-\mathrm{C} 4 & -24.2(4) \\ \mathrm{N} 1-\mathrm{N} 2-\mathrm{C} 6-\mathrm{N} 3 & -3.5(5) & \mathrm{C} 2-\mathrm{C} 3-\mathrm{C} 4-\mathrm{C} 5 & 38.1(3) \\ \mathrm{C} 7-\mathrm{N} 3-\mathrm{C} 6-\mathrm{S} 1 & 2.2(5) & \mathrm{C} 3-\mathrm{C} 4-\mathrm{C} 5-\mathrm{C} 1 & -36.6(3) \\ \mathrm{C} 7-\mathrm{N} 3-\mathrm{C} 6-\mathrm{N} 2 & -179.0(3) & \mathrm{N} 3-\mathrm{C} 7-\mathrm{C} 8-\mathrm{C} 9 & 113.4(6) \\ \mathrm{C} 6-\mathrm{N} 3-\mathrm{C} 7-\mathrm{C} 8 & 80.2(5) & & \end{array}$

Hydrogen-bond geometry $\left(\AA,{ }^{\circ}\right)$

\begin{tabular}{lllll}
\hline$D-\mathrm{H} \cdots A$ & $D-\mathrm{H}$ & $\mathrm{H} \cdots A$ & $D \cdots A$ & $D-\mathrm{H} \cdots A$ \\
\hline $\mathrm{N} 2-\mathrm{H} 2 C \cdots \mathrm{N} 1^{\mathrm{i}}$ & 0.91 & 2.29 & $3.194(4)$ & 177 \\
$\mathrm{~N} 3-\mathrm{H} 3 C \cdots \mathrm{N} 1$ & 0.91 & 2.25 & $2.642(4)$ & 106 \\
$\mathrm{~N} 3-\mathrm{H} 3 C \cdots \mathrm{S} 1^{\mathrm{ii}}$ & 0.91 & 2.49 & $3.310(3)$ & 151 \\
$\mathrm{C} 3-\mathrm{H} 3 A \cdots \mathrm{S} 1^{\mathrm{iii}}$ & 0.99 & 2.86 & $3.663(4)$ & 139
\end{tabular}

Symmetry codes: (i) $-y+1, x, z+1 / 4$; (ii) $y,-x+1, z-1 / 4$; (iii) $-y, x, z+1 / 4$. 